University of New Hampshire

University of New Hampshire Scholars' Repository

Space Science Center

Institute for the Study of Earth, Oceans, and

Space (EOS)

1994

\title{
Recent results from COMPTEL observations of Cygnus X-1
}

\author{
Mark L. McConnell \\ University of New Hampshire - Main Campus, mark.mcconnell@unh.edu \\ D J. Forrest \\ University of New Hampshire - Main Campus \\ James M. Ryan \\ University of New Hampshire, James.Ryan@unh.edu \\ W Collmar \\ Max-Planck-Institut für extraterrestriche Physik \\ V Schonfelder \\ Max-Planck-Institut für extraterrestrische Physik
}

See next page for additional authors

Follow this and additional works at: https://scholars.unh.edu/ssc

Part of the Astrophysics and Astronomy Commons

\section{Recommended Citation}

Recent results from COMPTEL observations of Cygnus X-1 McConnell, M. and Forrest, D. and Ryan, J. and Collmar, W. and Schönfelder, V. and Steinle, H. and Strong, A. and van Dijk, R. and Hermsen, W. and Bennett, K. and Much, R., AIP Conference Proceedings, 304, 230-234 (1994), DOl:http://dx.doi.org/ $10.1063 / 1.45624$

This Conference Proceeding is brought to you for free and open access by the Institute for the Study of Earth, Oceans, and Space (EOS) at University of New Hampshire Scholars' Repository. It has been accepted for inclusion in Space Science Center by an authorized administrator of University of New Hampshire Scholars' Repository. For more information, please contact Scholarly.Communication@unh.edu. 


\section{Authors}

Mark L. McConnell, D J. Forrest, James M. Ryan, W Collmar, V Schonfelder, H Steinle, A W. Strong, R VanDijk, W Hermsen, K Bennett, and R Much 


\section{AIP $\mid$ proceedings}

\section{Recent results from COMPTEL observations of Cygnus X1}

M. McConnell, D. Forrest, J. Ryan, W. Collmar, V. Schönfelder, H. Steinle, A. Strong, R. van Dijk, W. Hermsen, K. Bennett, and R. Much

Citation: AIP Conference Proceedings 304, 230 (1994); doi: 10.1063/1.45624

View online: $\mathrm{http}: / / \mathrm{dx}$.doi.org/10.1063/1.45624

View Table of Contents:

http://scitation.aip.org/content/aip/proceeding/aipcp/304?ver=pdfcov

Published by the AIP Publishing

Articles you may be interested in

COMPTEL observations of gammaray flares in October 1991

AIP Conf. Proc. 294, 55 (1994); 10.1063/1.45200

Comptel gammaray observations of the quasars CTA 102 and 3C 454.3

AIP Conf. Proc. 304, 644 (1994); 10.1063/1.45665

OSSE observations of blazars

AIP Conf. Proc. 304, 587 (1994); 10.1063/1.45657

BATSE observations of Cygnus X1

AIP Conf. Proc. 304, 220 (1994); 10.1063/1.45622

COMPTEL observations of Cygnus X1

AIP Conf. Proc. 280, 335 (1993); 10.1063/1.44142 


\title{
RECENT RESULTS FROM COMPTEL OBSERVATIONS OF CYGNUS X-1
}

\author{
M. McConnell, D. Forrest, J. Ryan \\ Space Science Center, University of New Hampshire, Durham, NH 03824 \\ W. Collmar, V. Schönfelder, H. Steinle, A. Strong \\ Max Planck Institute for Extraterrestrial Physics, 8046 Garching, FRG \\ R. van Dijk*, W. Hermsen \\ SRON-Leiden, 2300 RA Leiden, The Netherlands \\ K. Bennett, R. Much \\ Astrophysics Division, ESTEC, 2200 AG Noordwijk, The Netherlands
}

\begin{abstract}
The COMPTEL experiment on the Compton Gamma-Ray Observatory (CGRO) has now observed Cyg X-1 on four separate occasions during phase 1 and phase 2 of its orbital mission (April, 1991 to August, 1993). Here we report on the results of the latest analysis of these data, which provide a spectrum extending to energies greater than $2 \mathrm{MeV}$. A spectral analysis of these data, in the context of a classical Comptonization model, indicates an electron temperature much higher than previous hard $\mathrm{X}$-ray measurements would suggest ( $200 \mathrm{keV}$ vs $60-80 \mathrm{keV}$ ). This implies either some limitations in the standard Comptonization model and/or the need to incorporate a reflected component in the hard X-ray spectrum. Although significant variability near $1 \mathrm{MeV}$ has been observed, there is no evidence for any 'MeV excess'.
\end{abstract}

\section{INTRODUCTION}

The COMPTEL experiment is designed to measure $\gamma$-radiation in the range from 0.75-30 MeV (Schönfelder et al. 1993). This is a particularly interesting energy range for the black-hole candidate Cyg X-1. In recent years, data from several experiments have suggested that the single-temperature Sunyaev-Titarchuk Comptonization model may be inadequate to explain the hard X-ray data between several hundred $\mathrm{keV}$ and $1 \mathrm{MeV}$ (e.g., Grebenev et al. 1993; Grabelsky et al. 1993). In addition, some observers have also reported the detection of a significant spectral hardening (to about $\mathrm{E}^{-1}$ ) at energies above 1 MeV (e.g., Baker et al. 1973; Ling et al. 1987; McConnell et al. 1989). The measured spectra of this 'MeV excess' are clearly inconsistent with a simple extrapolation of the hard $X$-ray spectrum. The reported flux levels are more than two orders of magnitude above the COMPTEL detection threshold, suggesting that COMPTEL would be capable of studying such emission in detail. Here we shall present the latest results from the analysis of the COMPTEL data and compare these results with other measurements at these energies.

\section{OBSERVATIONS AND ANALYSIS}

A total of roughly seven weeks of observation time on Cyg X-1 has been collected from six separate viewing periods during the first two phases ( 28 months) of the CGRO mission (see table). (Note that periods 203.0 - 203.6 represented only a change in the spacecraft orientation about its $\mathrm{z}$-axis, rather than a change in the actual pointing direction. So in the present analysis, we treat these as a single observation.) In all cases, Cyg X-1 was within $15^{\circ}$ of the COMPTEL pointing direction.

* also Astronomical Institute "Anton Pannekoek", University of Amsterdam 
M. McConnell et al. 231

\begin{tabular}{ccc}
\hline \hline $\begin{array}{c}\text { Observation } \\
\text { Period }\end{array}$ & Dates & $\begin{array}{c}\text { Angle from z- } \\
\text { axis }\end{array}$ \\
\hline 2.0 & May 30 - June 8, 1991 & $2^{\circ}$ \\
7.0 & August 8-15, 1991 & $11^{\circ}$ \\
203.0 & December 1-8, 1992 & $7^{\circ}$ \\
203.3 & December 8-15, 1992 & $7^{\circ}$ \\
203.6 & December 15-22, 1992 & $7^{\circ}$ \\
212.0 & March 9-23,1993 & $15^{\circ}$ \\
\hline \hline
\end{tabular}

The present analysis follows that described by McConnell et al. (1994). In particular, we have analyzed the data in six separate energy intervals: $0.75-0.85 \mathrm{MeV}, 0.85$ 1.0 MeV, 1.0-2.0 MeV, 2.0-5.0 MeV, 5.0-10.0 MeV, and 10.0-30.0 MeV. For each observation, the data in each of these six energy intervals are analyzed using a maximum likelihood approach (de Boer et al. 1992). This analysis incorporates an instrumental point-spread-function (PSF) which is generated on the basis of an assumed source spectrum. In the study of Cyg X-1, we generally employ PSFs based on a Wien spectrum, which represents the high energy limit of the Sunyaev-Titarchuk Comptonization model (Sunyaev and Titarchuk 1980). For the datapoints above $5 \mathrm{MeV}$ (where the Wien spectrum falls off very steeply), the analysis has employed PSFs based on an $\mathrm{E}^{-2}$ power law spectrum.

The analysis of each energy interval results in a flux value corresponding to that energy range. Once the results from each energy range are available, a photon spectrum can be compiled. A fit of the data in photon space is used to derive a corresponding electron temperature. A self-consistency check can then be made by ensuring that the electron temperature used in deriving the PSFs corresponds to that derived from the spectral fit. Here, several different PSFs (corresponding to diferent values of $k T$ ) were used. The final spectral results used a PSF for $\mathrm{kT}=200 \mathrm{keV}$.

\section{RESULTS}

We have previously reported on the results from an analysis of the phase 1 data (periods 2.0 and 7.0; McConnell et al. 1993, 1994). These data indicated a firm detection of the source up to $2 \mathrm{MeV}$. The complete analysis of all four individual observations shows very similar spectra in all cases. Indeed, the derived electron temperatures are all in the range of $180-220 \mathrm{keV}$. Our data also show that the flux around $1 \mathrm{MeV}$ is variable on a time scale of several weeks, as can be seen in Figure 1. Although the flux levels for periods 2.0, 203 and 212.0 are roughly equivalent, the flux level for period 7.0 is about a factor of two higher. This variation is roughly similar to that observed by BATSE near 100 $\mathrm{keV}$ (Ling et al. 1994).

In addition to analyzing the data from the individual observation periods, we have combined all seven weeks of data into one single spectrum. This spectrum is shown in Figure 2 along with other representative spectra from previous experiments. The electron temperature derived from these data is $\mathrm{kT}=190 \pm 20 \mathrm{keV}$. One interesting aspect of the COMPTEL spectrum is that the $2-5 \mathrm{MeV}$ datapoint is significant at the $3.5 \sigma$ level. In order to confirm this detection, it is instructive to look at the imaged data. Figure 3 shows the imaged data from the $0.75-1.0 \mathrm{MeV}$ energy band. A clear signal (with $12 \sigma$ significance) is seen at the location of Cyg X-1. A similar image for the 2-5 MeV interval also shows a point-like feature which is consistent with the location of Cyg X-1. This confirms that we have indeed detected Cyg X-1 at energies above $2 \mathrm{MeV}$. However, there is no evidence in these data for any significant hardening of the spectrum at or above $1 \mathrm{MeV}$. 


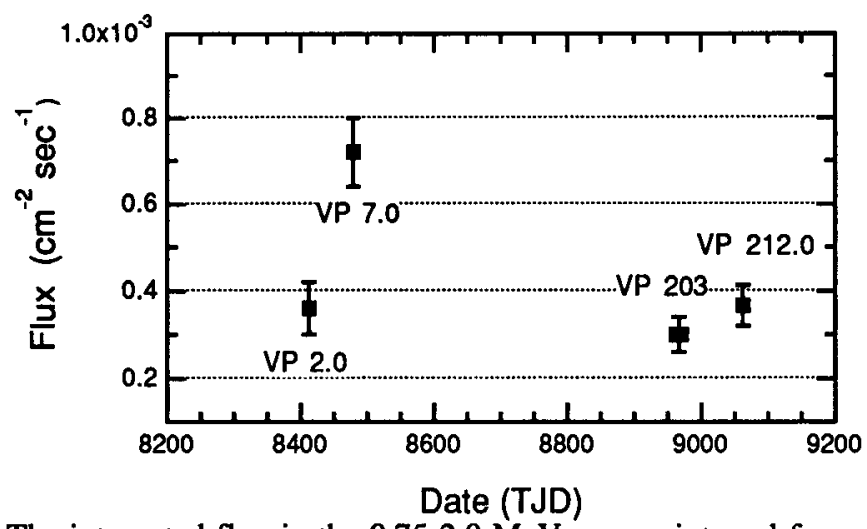

Fig. 1. The integrated flux in the $0.75-2.0 \mathrm{MeV}$ energy interval for each of the four observations of Cyg X-1. The higher flux level in viewing period 7.0 is clearly seen.

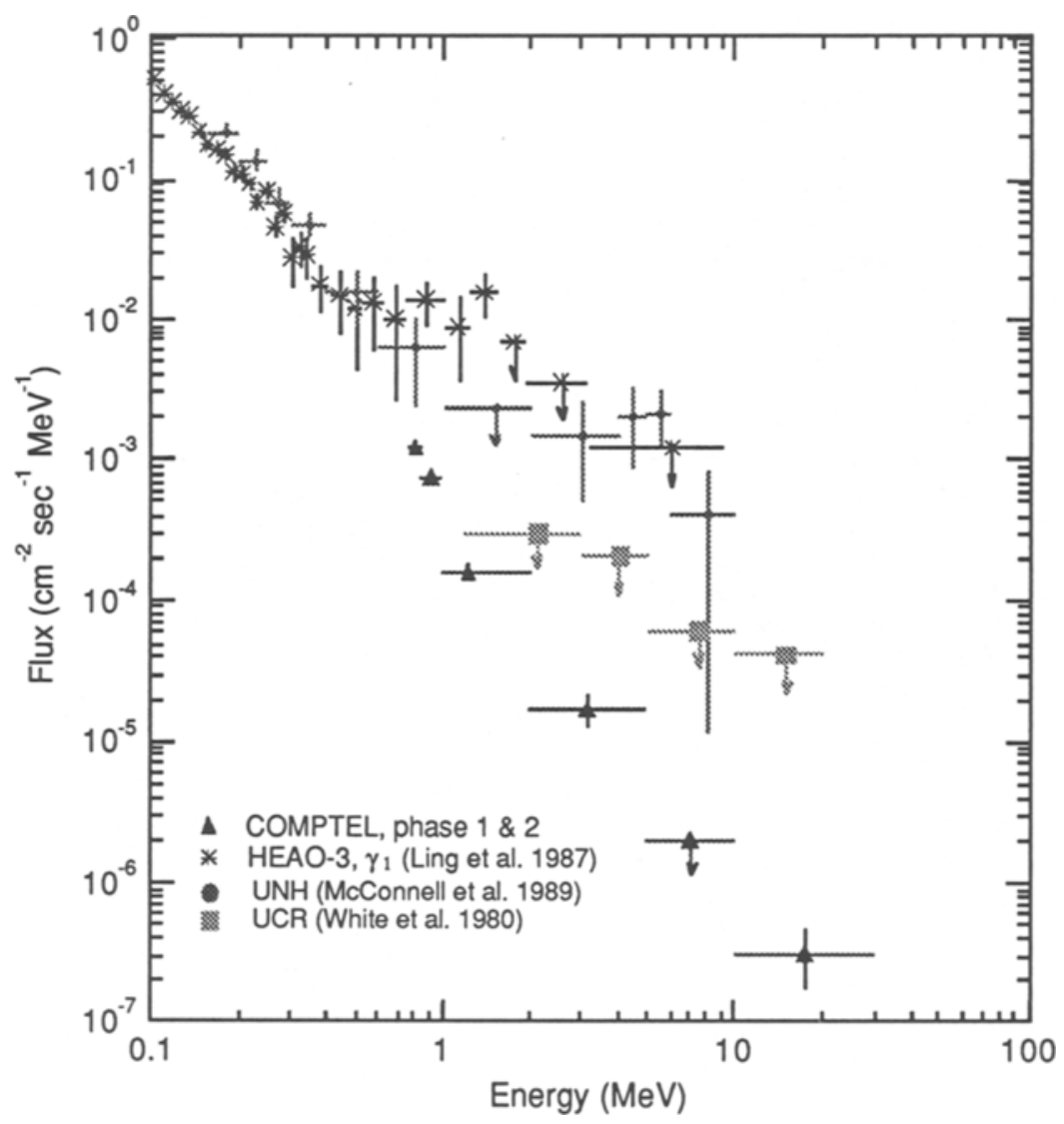

Fig. 2. The average spectrum of Cyg X-1 observed by COMPTEL from observations obtained during phase 1 and phase 2 of the CGRO mission. 


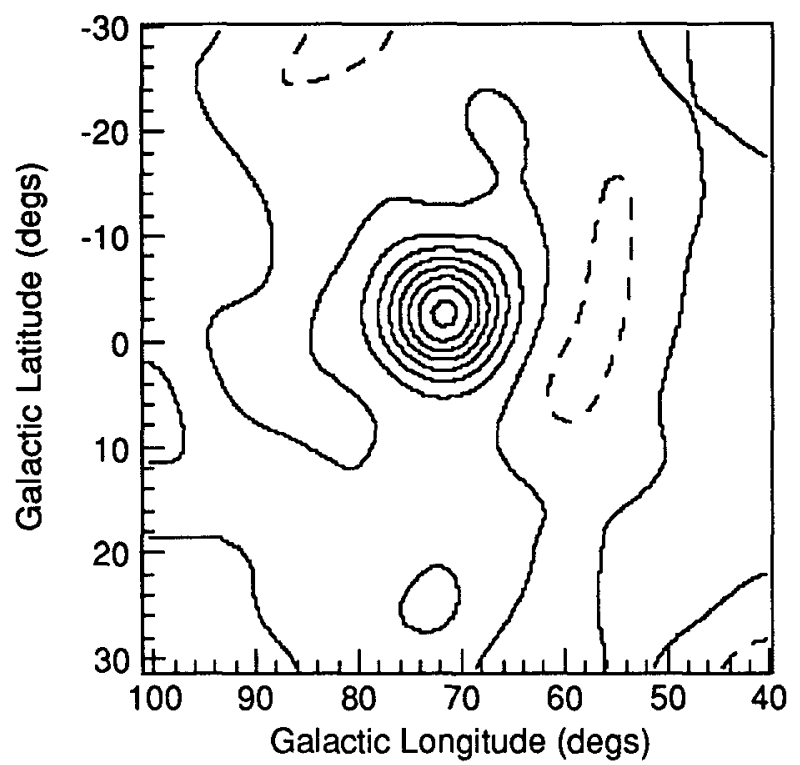

Fig. 3. Maximum likelihood image of the Cygnus region for the $0.75-1.0 \mathrm{MeV}$ energy range derived from seven weeks of data collected during phase 1 and phase 2 of the CGRO mission. The response from Cyg X-1 (at $l=71.3^{\circ}, b=3.1^{\circ}$ ) stands out clearly. (The likelihood contours start at a level of -30 with a stepsize of 15.)

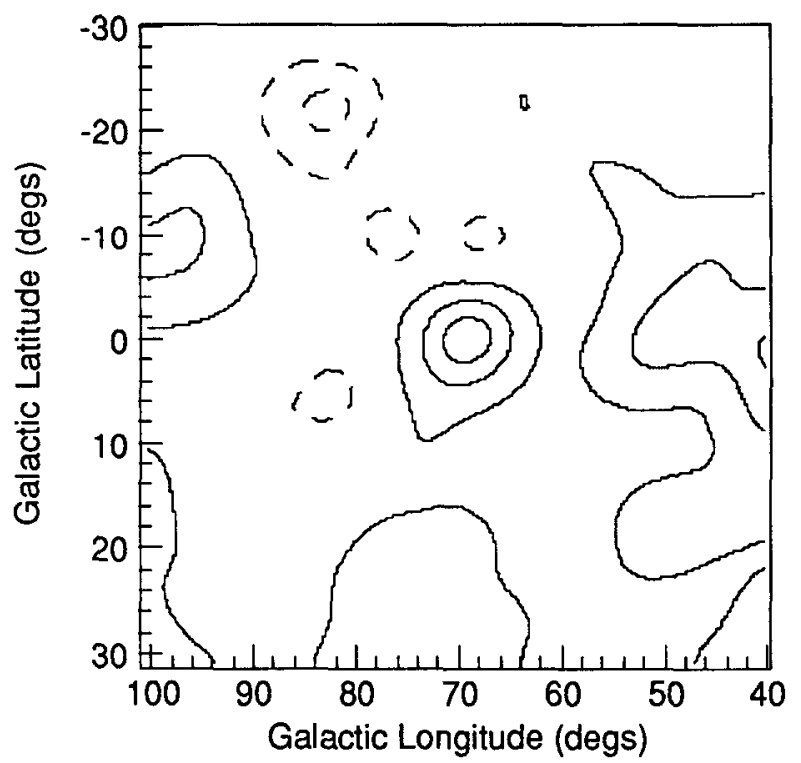

Fig. 4. Same as in Figure 3, but now for the energy interval 2.0-5.0 MeV. A response which is consistent with the location of Cyg X-1 is still seen. The significance of this detection is at the $3.5 \sigma$ level. (The likelihood contours start at a level of -30 with a stepsize of 8 .) 


\section{DISCUSSION}

We have now extended the measurement of the Cyg X-1 spectrum to energies upwards of $2 \mathrm{MeV}$. Variability near $1 \mathrm{MeV}$ on a time scale of weeks has been observed. This variability, to first order, appears to be correlated with the variability near $100 \mathrm{keV}$.

The electron plasma temperature which we derive for a Wien-type spectrum (kT $\sim 190 \mathrm{keV}$ ) differs considerably from typical temperatures measured at hard $\mathrm{X}$-ray energies $(\mathrm{kT} \sim 60 \mathrm{keV})$. This conflict would suggest that our standard picture of the Cyg X-1 system needs to be revised. We suggest here two possible explanations which may serve to resolve this discrepancy in the derived temperatures.

First, it should be noted that the standard spectral modeling of Cyg X-1 (as well as many other accreting X-ray sources) is based on the Comptonization model of Sunyaev and Titarchuk (1980). Recent observations of Cyg X-1 by both OSSE (Grabelsky et al. 1993) and SIGMA (Grebenev et al. 1993) have already suggested that this model is inadequate to explain the broad-band observations (i.e., from $<100 \mathrm{keV}$ up to $1 \mathrm{MeV}$ ). Typically, a twotemperature Comptonization model provides a better fit to the data. This has led some to conclude that the underlying assumptions of the analytical Sunyaev-Titarchuk model are not valid in the case of Cyg X-1. For example, Grebenev et al. (1993) have used more detailed Monte Carlo modeling of the Comptonization process to fit the observed spectrum.

Second, recent modeling of the X-ray emission (Haardt et al. 1993) has suggested that any comprehensive model of Cyg X-1 must include a component which represents the reflection of hard X-rays off a cooler optically-thick accretion disk. This is in direct analogy to the modeling of AGN spectra. Haardt et al. (1993) have employed such a model in their analysis of EXOSAT data to suggest that the hard X-ray emission comes predominantly from an accretion disk corona with $\mathrm{kT}$ in the $130-200 \mathrm{keV}$ range. The reflection of this hard X-ray flux off the optically thick accretion disk leads to a distortion of the hard X-ray spectrum which can explain the measured values of $\sim 60 \mathrm{keV}$. The very high energy threshold of COMPTEL $(750 \mathrm{keV})$ would make it relatively immune to the effects of any reflected component. Indeed, it is interesting to note that the coronal temperature implied by the EXOSAT data is roughly similar to that suggested by the COMPTEL data.

This work was supported (in part) by NASA under grant NAS5-26645 and by DARA under grant 50 QV 90968.

\section{REFERENCES}

Baker, R.E., et al. 1973, Nature Phys. Sci., 245, 18.

de Boer, H., et al. 1992, in 'Data Analysis in Astronomy IV' (New York: Plenum Press), p. 241.

Done, C., Mulchaey, J. S., Mushotzky, R. F., \& Arnaud, K. A. 1992, ApJ, 395, 275.

Grabelsky, D. A., et al. 1993, in Compton Gamma-Ray Observatory (AIP Conf. Proc. 280), eds. Friedlander et al. (New York: American Institute of Physics), p. 345.

Grebenev, S., et al. 1993, A\&AS, 97, 281.

Haardt, F., Done, C., Matt, G., \& Fabian, A. C. 1993, ApJ, 411, L95.

Ling, J. C., et al. 1987, ApJ, 321, L117.

Ling, J.C., et al.. 1994, these proceedings.

McConnell, M. L., et al. 1989, ApJ, 343, 317.

McConnell, M. L., et al. 1993, in Compton Gamma-Ray Observatory (AIP Conf. Proc. 280), eds. Friedlander et al. (New York: American Institute of Physics), p. 335.

McConnell, M. L., et al. 1994, ApJ, in press.

Schönfelder, V., et al. 1993, ApJS, 86, 629.

Sunyaev, R. A. \& Titarchuk, L. G. 1980, A\&A, 86, 121.

White, R. S., et al. 1980, Nature, 284, 608. 